\title{
Surgical management of an extensive spinal epidural abscess: illustrative cases
}

\author{
Aleksey Eroshkin, MD, PhD, ${ }^{1}$ Dmytro Romanukha, MD, ${ }^{1}$ and Serhiy Voitsekhovskyi, MD² \\ 'Department of Neurosurgery, Central Hospital of Ministry of Internal Affairs of Ukraine (Central Police Hospital), Kyiv, Ukraine; and ${ }^{2}$ Bogomolets National Medical University, \\ Kyiv, Ukraine
}

\begin{abstract}
BACKGROUND Extensive spinal epidural abscesses (SEAs) occupying three or more spinal regions are rare. This study aimed to address the key dilemma of surgical treatment for holospinal epidural abscesses, i.e., to determine the required scope of surgery and minimize surgical trauma with adequate purulent drainage.
\end{abstract}

OBSERVATIONS Two patients with extensive SEAs were treated at the Neurosurgery Department of the Central Hospital of Ministry of Internal Affairs of Ukraine from 2018 to 2020. Both patients had a neurological deficit and general inflammatory response syndrome. Spinal magnetic resonance examinations were performed, showing that the first and second patients had extensive SEAs at T11/S1 and C2/L1, respectively. Both underwent minimally invasive abscess drainage via intra- and translaminar access at the most caudal point using an epidural silicone catheter in the cranial direction along the entire length of the abscess.

LESSONS To achieve the key goal of extensive SEA treatment, i.e., to prevent the development of a persistent neurological deficit, immediate effective spinal canal decompression should be performed. Access method and scope should meet the requirements of spinal canal decompression and purulent content aspiration to the greatest possible extent while inducing minimal trauma.

https://thejns.org/doi/abs/10.3171/CASE2050

KEYWORDS extensive spinal abscess; holospinal abscess; epidural abscess; epidural drainage

Spinal epidural abscess (SEA) is defined as the accumulation of purulent contents in the epidural space of the spinal canal, causing spinal medullary and root ischemia via compression, resulting in persistent neurological disorders and generalized inflammation. ${ }^{1}$ The incidence of SEA is estimated at 0.2-2 per 10,000 hospitalized patients. ${ }^{2}$ More than 1,000 clinically recorded patients with treated SEA over the past 50 years have been reported. ${ }^{3}$ This information is sufficient to determine the etiology, pathogenesis, clinical presentation, and effective diagnostic methods. However, the standard optimal treatment techniques and methods remain to be established-in the early 1900s, SEA was a fatal disease, and by the end of the century, mortality caused by SEA was reduced to $15 \%-20 \%{ }^{3}$ More than half of the patients continue to have neurological deficits after treatment. ${ }^{3-5}$ Successful conservative antibacterial SEA treatment has already been reported; however, neurological deficits, coexisting chronic diseases, and methicillin-resistant Staphylococcus aureus (MRSA) infection are negative prognostic criteria that require surgical intervention. ${ }^{6,7}$ Over the past 50 years, the incidence of epidural abscesses has doubled 2,3 because of the increased number of invasive methods for the treatment of spinal diseases, the increased number of chronic diseases in older age groups, the spread of intravenous drug use, and the development of more effective diagnostic methods (e.g., computed tomography $[\mathrm{CT}]$ and magnetic resonance imaging $[\mathrm{MRI}]){ }^{2,7-9}$

According to statistics, more than $70 \%$ of SEA cases arise from localized or systemic inflammatory diseases (e.g., regional abscesses and spondylodiscitis) or immunodeficiency diseases (e.g., human immunodeficiency virus), and approximately $22 \%$ occur postoperatively. ${ }^{4}$ Approximately $70 \%$ of cases are caused by methicillin-susceptible Staphylococcus aureus (MSSA) and MRSA, which develop more frequently among patients with spinal or cardiovascular implants.

The following pathogens have also been found: Staphylococcus epidermidis, Escherichia coli, and Pseudomonas aeruginosa, among others. ${ }^{4}$

ABBREVIATIONS $\mathrm{CBC}=$ complete blood count; $\mathrm{CT}=$ computed tomography; $\mathrm{ESR}=$ erythrocyte sedimentation rate; $\mathrm{MRI}=$ magnetic resonance imaging; $\mathrm{MRSA}=$ methicillin resistant Staphylococcus aureus; MSSA = methicillin-susceptible Staphylococcus aureus; SEA = spinal epidural abscess; VAS = visual analog scale.

INCLUDE WHEN CITING Published January 11, 2021; DOI: 10.3171/CASE2050.

SUBMITTED October 3, 2020. ACCEPTED October 30, 2020.

(c) 2021 The authors, CC BY-NC-ND 4.0 (http://creativecommons.org/licenses/by-nc-nd/4.0/) 
SEAs have been classified into two groups depending on their extension: local, covering up to two spinal regions, and extensive (holospinal or multiregional), covering three or more regions. ${ }^{10}$

The latter is rare, comprising $<25$ holospinal abscess cases over the past 25 years. ${ }^{11}$ Only $1 \%$ of $>900$ patients with an SEA in a large metaanalysis had a holospinal abscess that covered the cervical-thoraciclumbar region. ${ }^{4}$ SEA may also predominantly extend along the front surface of the epidural space, pushing the medulla to the posterior ventral abscess and vice versa, and can be located behind the dural sac, i.e., the dorsal SEA. In the scientific literature, most authors prefer active surgical techniques for the treatment of extensive abscess. The methods applied include mono- and multilevel laminectomy, followed by epidural drainage installation or percutaneous drainage injection under CT control with further purulent content aspiration and lavage. ${ }^{1,2,11}$ The key dilemma of surgical holospinal SEA treatment is to determine the required scope of surgery and minimize surgical trauma with adequate suppurative drainage.

Extensive multilevel laminectomies may be impossible for several reasons: serious or critical patient condition, risks of mechanical spinal instability, increased surgical duration, and major blood loss, among others.

In this report, we present the treatment methods used for two patients with extensive SEA indicated for surgical drainage due to the increased rate of clinical symptoms and extensive process.

We have detailed the method used, reviewed its advantages, and compared it with other methods used in similar clinical conditions.

The SEA was accessed from its most caudal point. An interlaminectomy or laminectomy was performed at the distal pole of the abscess, followed by epidural drain installation (Fig. 1).

Intraoperatively, a 3-mm-diameter perforated silicone drainage catheter was used and introduced in the cranial direction along the entire SEA length.

The cranial drainage catheter was restricted to the abscess wall or to when it reached an obstacle while attempting to advance it.

A syringe was attached to the distal end of the catheter, and the abscess contents were aspirated as the catheter progressed, with part of the abscess evacuated through aspiration for further microbiological testing.

After sanitation and irrigation using an antiseptic solution of $0.2 \mathrm{mg} / \mathrm{ml}$ decamethoxin (Yuria-Pharm, Kiev, Ukraine) and saline in a ratio of 1:2, the drainage catheter was mandatorily extracted through a counteropening of at least $5 \mathrm{~cm}$, lateral to the right or left of the cut, and stitched to the skin.

If spondylodiscitis was the cause of an extensive SEA, an extended interlaminectomy was performed in addition to the drainage at this level, and the primary focus (spondylodiscitis) was sanitized.

\section{Illustrative Cases}

\section{Case 1}

In November 2018, a 49-year-old man was hospitalized due to intense pain in the lumbar spine that radiated to both lower limbs, on the posterior lateral surface of the thighs and tibia, with a visual analog scale (VAS) score of $8,{ }^{12}$ progressive lower limb weakness, a temperature of up to $39^{\circ} \mathrm{C}$, and general weakness. During the preceding month, the patient had experienced a low-grade fever of an unknown cause. One week prior to treatment, the patient had experienced lumbodynia with progressing intensity that limited the range of motion in the lumbar spine, radiating pain to the lower extremities, and causing weakness.

Thoracolumbalis, hypesthesia in the lower extremities at L5, bilateral dermatome, and paraparesis at $L 4 / 5$ were found during the

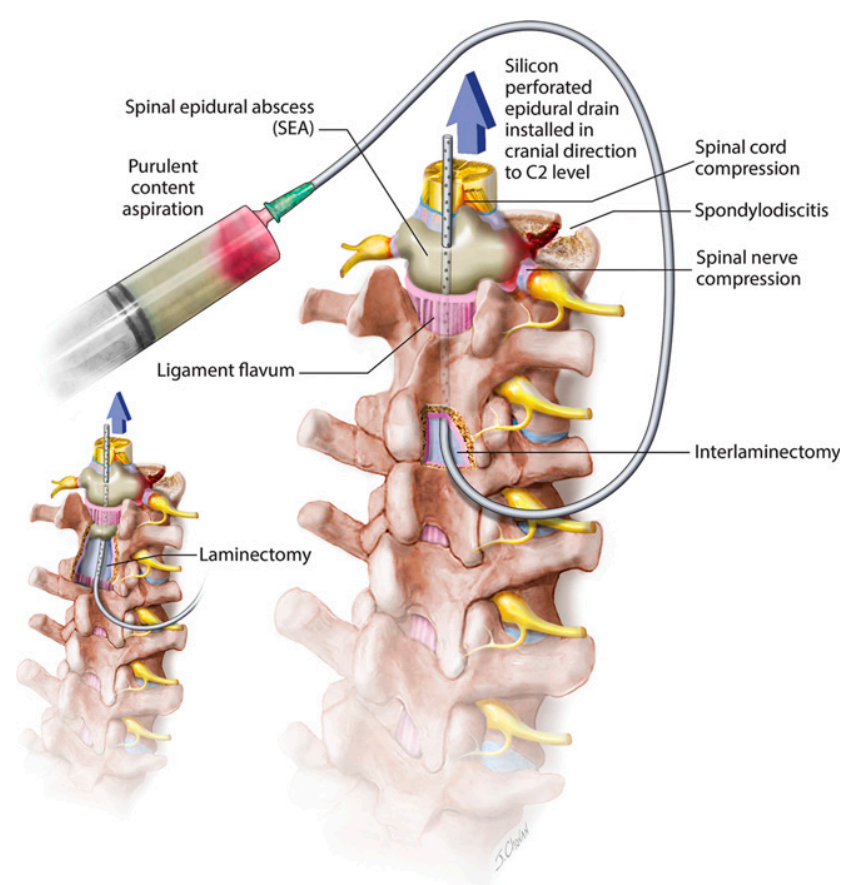

FIG. 1. Schematic view of epidural space drainage.

examination. The patient also had incontinence-related pelvic organ dysfunction. His blood test results were as follows: complete blood count $(\mathrm{CBC})$ showed leukocytosis, $19 \times 10^{9}$, and an increased erythrocyte sedimentation rate (ESR), $54 \mathrm{~mm} / \mathrm{hr}$. Thoracic and lumbar spine MRI identified an SEA at T11/S1, discitis at L5/S1, and signs of retrolisthesis at L5 (Fig. 2). The caudal pole of the abscess at L5/S1 was applied. The drainage catheter was inserted into the epidural space in the cranial direction along the entire SEA length.

Active content aspiration was performed with a syringe, followed by passive drainage.

Bilateral interlaminar decompression at $L 4 / 5$ and posterior spondylosyndesis using the transpedicular fixation system at L4/L5/ S1 were also performed to decompress the spinal canal, fully sanitize the site, and stabilize the provoked spinal motor segment (Fig. 3A and $\mathrm{B}$ ).

No causative pathogen was found in a blood culture test, and MSSA was identified by testing contents isolated from the epidural space. For 10 postoperative days, $1 \mathrm{~g}$ of ceftriaxone and $1 \mathrm{~g}$ of meropenem was intravenously administered twice a day. Subsequent antibacterial therapy with $400 \mathrm{mg}$ of oral moxifloxacin once a day was administered for 2 months. On the second postoperative day, the pain syndrome improved (VAS score $=3$ ) and his body temperature decreased to subfebrile. The drainage catheter was removed on day 3. On day 12, general inflammatory and focal symptoms regressed.

MRI (Fig. 3C) and follow-up examination at 6 months showed that the patient's state was satisfactory; his tendon and periosteal reflexes of the lower limbs were active and symmetrical, and he had no motor deficit or sensitivity disorder.

\section{Case 2}

The second case was that of a 58-year-old man who had complained of acute spinal pain, primarily thoracic and cervical, radiating to 

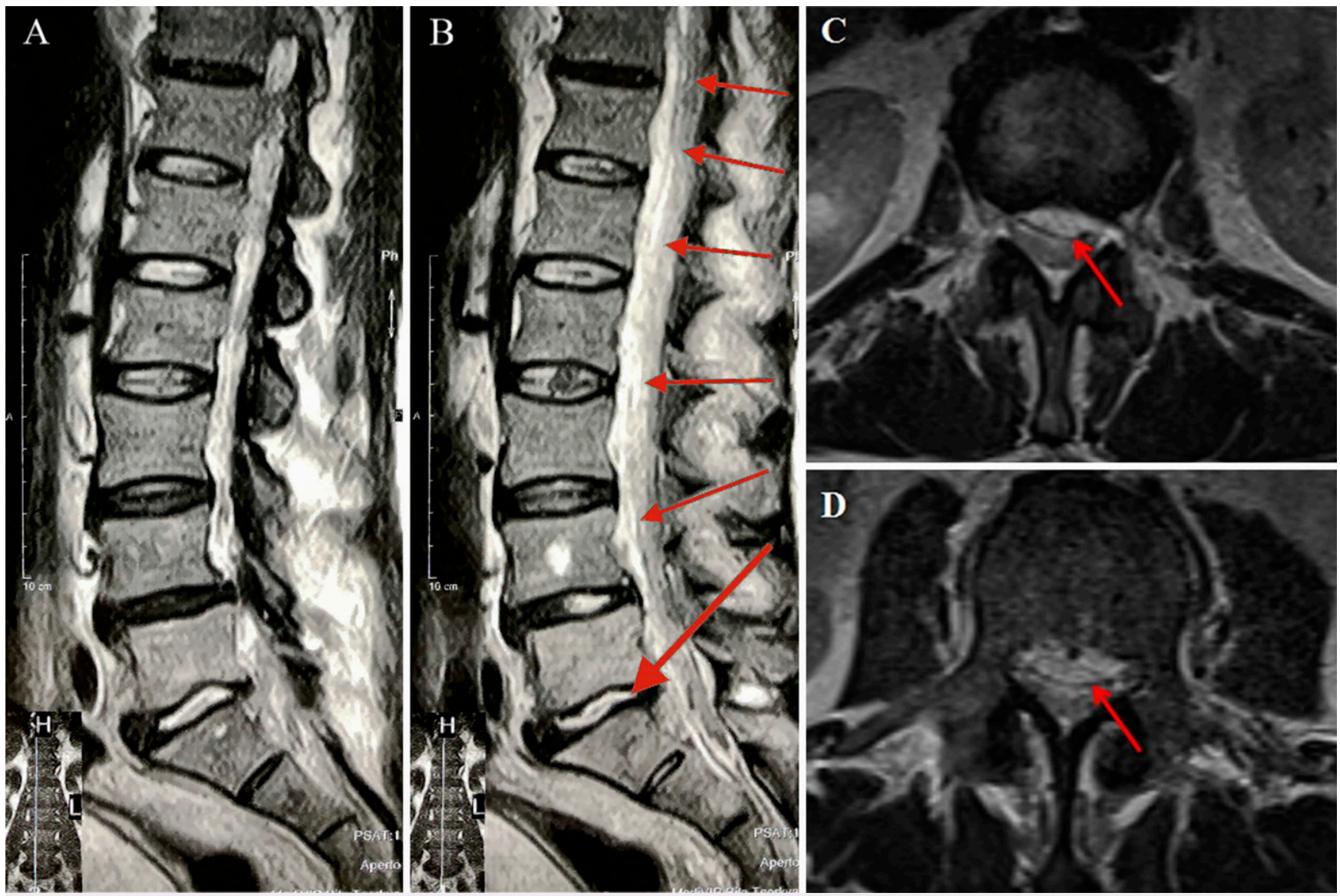

FIG. 2. Preoperative MRI in case 1. A: Sagittal plane, retrolisthesis at the $L 5$ vertebra on the background of $L 5 / S 1$ discitis. B: Sagittal plane, anterior extensive SEA (small arrows) at T11/S1 on the background of L5/S1 discitis (large arrow). C: Axial plane, ventral compression of SEA dural sac at T12/L1. D: Axial plane, ventral compression of SEA dural sac at L3 vertebra.

the upper limbs (VAS score $=6$ ), accompanied by limb weakness and a febrile temperature (up to $38.5^{\circ} \mathrm{C}$ ) in January 2020 . Symptoms acutely developed 2 days before presentation. The examination revealed weakened tendon and periosteal reflexes of the upper and lower extremities, lower extremity strength of $3.5 / 5$, upper extremity strength of $4 / 5$, hypesthesia from $\mathrm{C} 3$, dermatome, and cervicothoracic syndrome.

His blood test results were as follows: $\mathrm{CBC}$ showed leukocytosis, $26 \times 10^{9}$, and increased ESR, $72 \mathrm{~mm} / \mathrm{hr}$.

MRI confirmed an extensive SEA at C2/L1 on the spondylodiscitis background at the L1/2 segment (Fig. 4). The patient had type 2 diabetes mellitus. An extended laminectomy of the L1 vertebra was performed for adequate access. The drainage catheter was inserted in the cranial direction up to $\mathrm{C} 2$ along the entire length of the abscess, which was successfully drained. MRSA was cultured from the blood and aspirate. A follow-up CT of the drainage location in the epidural space was performed (Fig. 5). Combination antibiotic therapy was administered as follows: $1 \mathrm{~g}$ of ceftriaxone and $1 \mathrm{~g}$ of vancomycin were intravenously administered twice a day and once a day, respectively. Moxifloxacin $(400 \mathrm{mg}$ ) was administered once a day orally for the subsequent 2 months. Symptoms of cervicothoracic syndrome and generalized inflammation fully regressed on day 11 . The drainage catheter was removed on day 5 . The patient's condition at the 1-month
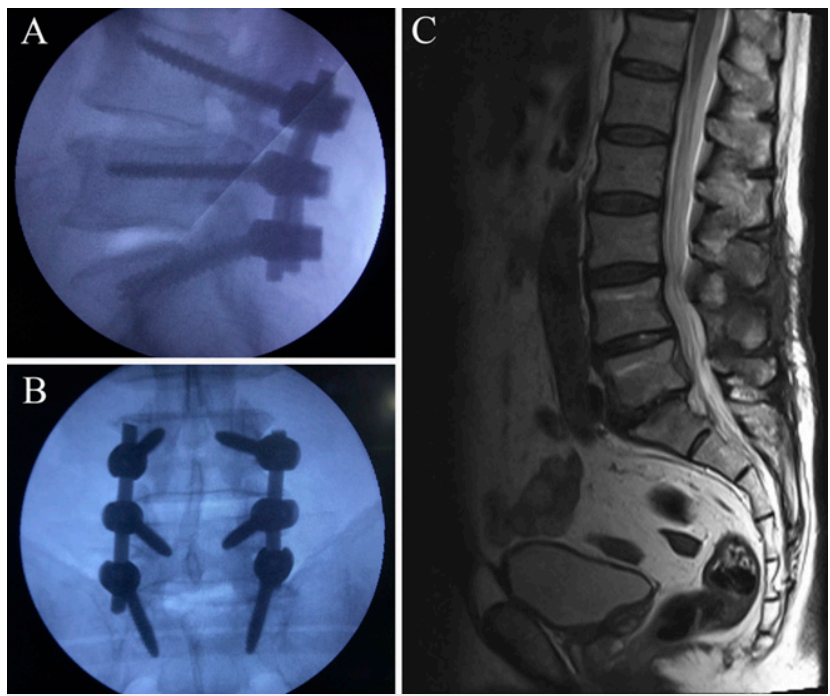

FIG. 3. Case 1. A, B: Follow-up radiography, lateral and anteroposterior views of L4/L5/S1 segment stabilization. C: MRI at 6 postoperative months. 

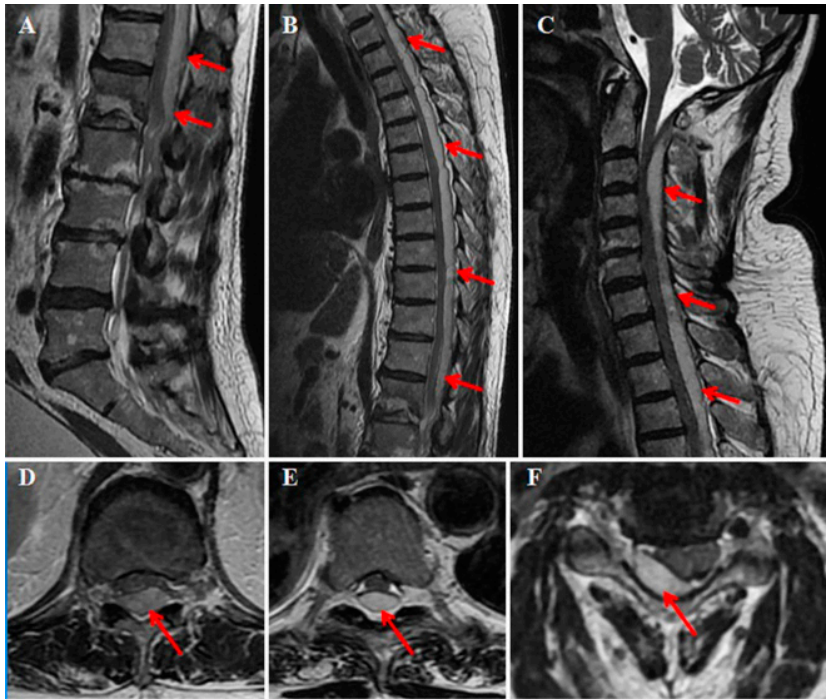

FIG. 4. Preoperative MRI in case 2. Sagittal plane, cervical (A), thoracic (B), and lumbosacral (C) spinal regions; the arrows indicate SEA spread on the background of $L 1 / 2$ spondylodiscitis. Axial plane, dorsal compression of the SEA dural sac at L1/2 (D), T7 (F), and $\mathrm{C} 3 / 4(\mathrm{E})$.

follow-up examination was satisfactory. He fully recovered from weakness in his extremities.

\section{Discussion}

\section{Observations}

The standard treatment for patients with an extensive SEA remains controversial. A successful case of conservative antibacterial treatment of holospinal SEA has been reported, ${ }^{10}$ and various percutaneous aspiration methods ${ }^{13,14}$ have been successfully used. However, the standard surgical approaches have not yet been established. Some authors suggest that selective laminectomies at the rostral and caudal poles of the abscess with subsequent drainage should be performed, ${ }^{15}$ whereas others have performed the so-called apical laminectomy on the natural spinal elevations, at the midcervical C3/5 level, the midthoracic T6/9 level, and the midlumbar L2/3 spinal segments. ${ }^{2}$ Laminectomy on the overlying T2/3 segments combined with hemilaminectomy on the underlying $L 1 / 2$ segments of the extensive SEA has also been reported. ${ }^{16}$

In addition, a thoracic spine laminoplasty ${ }^{17}$ and even a transoral dens extraction with partial C2 vertebral corpectomy and anterior arc removal of the $\mathrm{C} 1$ vertebra were performed to adequately drain the ventral holospinal SEA. ${ }^{18}$ Due to varying surgical scope, various epidural space drainage catheter types have been used. A Fogarty catheter, ${ }^{15}$ a $5-\mathrm{Fr}^{2}$ pediatric feeding tube, and soft silicone catheters for drainage have been used. ${ }^{16,19}$

Multilevel laminectomy may expose patients who often experience aggravating SEA-related somatic diseases due to various complications, such as mechanical instability, long surgical duration, or blood loss. However, medullar decompression and infection focus drainage should be performed.

In the case of progressive neurological deficit, most authors agree on surgical treatment for SEA.2,18,19 Instrumental fixation is rarely performed in an extensive SEA because the risk of implant
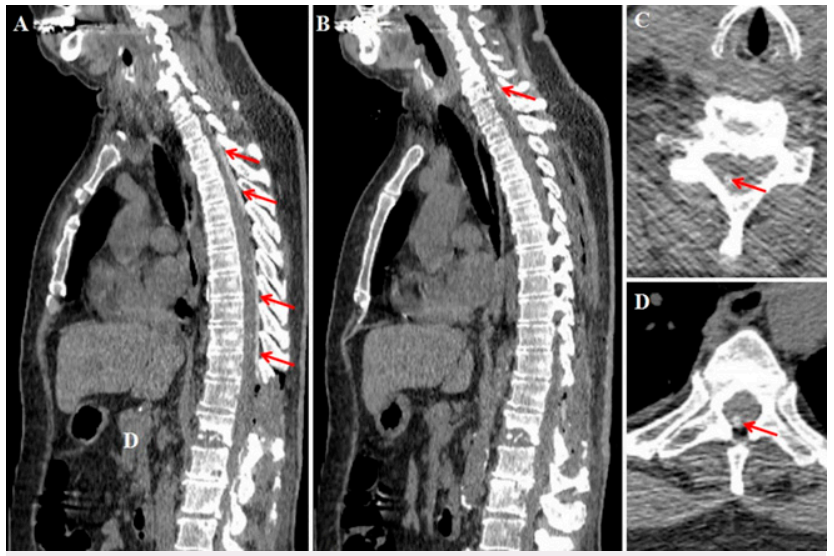

FIG. 5. Case 2. Postoperative CT, sagittal plane, epidural drainage imaging along the entire length of the thoracic spine $(\mathbf{A})$, and epidural drainage at C7 (B). Axial plane, epidural drainage imaging at the $\mathrm{C7}$ vertebra (C) and T7 vertebra (D).

contamination is not excluded. However, the positive role of fixation of the affected segment in spondylodiscitis has been increasingly discussed in the literature because focus drainage combined with immobilization facilitates early elimination of infection, early verticalization, and early patient activation with a significantly reduced risk of mechanical spinal instability. ${ }^{20-22}$ In both cases, holospinal epidural abscesses developed in patients with a history of spondylodiscitis. With regard to the extension, the first SEA was ventral and the other was dorsal. The affected segment, i.e., a retrolisthesis at the L5 vertebra in the first case, was unstable. Corresponding neurological and general inflammatory symptoms rapidly developed in both patients, which indicated a high risk of complications, including neurological deficits.

Therefore, when choosing treatment methods, conservative management was not performed because of the strong literature evidence of the need for urgent medullary and root decompression in an extensive SEA. $2,6,7$

A Fogarty catheter can be used to perform compression on the medulla, ${ }^{15}$ whereas 5 -Fr feeding tubes are, in our opinion, too thin and flexible, which can be a problem when placed in the epidural space, especially when granulation commissures or intersections are present or in the case of highly viscous purulence. We preferred the siliconeperforated epidural drainage catheter, which was rigid enough to run it along the entire abscess length. Perforations along the catheter increased the contact area with purulent content. Due to such drainage catheter installation, multilevel accesses were performed, depending on the requirements of spinal canal drainage and decompression. Consequently, this reduced surgical time and blood loss.

\section{Lessons}

It is difficult to establish the exact criteria for drainage method selection in an extensive SEA due to greatly varied SEA locations and the presence of coexisting diseases.

To achieve the key goal of treatment for extensive SEA, i.e., to prevent the development of persistent neurological deficits, immediate effective spinal canal decompression should be performed. Therefore, we recommend avoiding conservative treatment of extensive epidural abscesses. 
The access method and scope should meet the criteria of spinal canal decompression and purulent content aspiration to the greatest possible extent while inducing minimal trauma.

\section{References}

1. Rosc-Bereza K, Arkuszewski M, Ciach-Wysocka E, Boczarska-Jedynak M. Spinal epidural abscess: common symptoms of an emergency condition. A case report. Neuroradiol J. 2013;26(4):464-468.

2. Abd-El-Barr MM, Bi WL, Bahluyen B, et al. Extensive spinal epidural abscess treated with "apical laminectomies" and irrigation of the epidural space: report of 2 cases. J Neurosurg Spine. 2015;22(3):318-323.

3. Arko L 4th, Quach E, Nguyen V, et al. Medical and surgical management of spinal epidural abscess: a systematic review. Neurosurg Focus. 2014;37(2):E4.

4. Reihsaus $E$, Waldbaur $H$, Seeling W. Spinal epidural abscess: a meta-analysis of 915 patients. Neurosurg Rev. 2000;23(4): 175-205.

5. Sendi $P$, Bregenzer T, Zimmerli W. Spinal epidural abscess in clinical practice. QJM. 2008;101(1):1-12.

6. Kim SD, Melikian R, Ju KL, et al. Independent predictors of failure of nonoperative management of spinal epidural abscesses. Spine J. 2014;14(8):1673-1679.

7. Connor DE Jr, Chittiboina P, Caldito G, Nanda A. Comparison of operative and nonoperative management of spinal epidural abscess: a retrospective review of clinical and laboratory predictors of neurological outcome. J Neurosurg Spine. 2013;19(1): 119-127.

8. Stüer C, Stoffel M, Hecker J, et al. A staged treatment algorithm for spinal infections. J Neurol Surg A Cent Eur Neurosurg. 2013;74(2): 87-95.

9. Lener S, Hartmann S, Barbagallo GMV, et al. Management of spinal infection: a review of the literature. Acta Neurochir (Wien). 2018;160(3):487-496.

10. Killen MC, Hernandez M, Berg A, Bhatia C. Nonoperative management of a multi-regional epidural abscess with neurological dysfunction. Int J Spine Surg. 2015;9:E47.

11. Smith GA, Kochar AS, Manjila S, et al. Holospinal epidural abscess of the spinal axis: two illustrative cases with review of treatment strategies and surgical techniques. Neurosurg Focus. 2014;37(2):E11.

12. Delgado DA, Lambert BS, Boutris $\mathrm{N}$, et al. Validation of digital visual analog scale pain scoring with a traditional paper-based visual analog scale in adults. J Am Acad Orthop Surg Glob Res Rev. 2018;2(3):e088.

13. Lyu RK, Chen CJ, Tang LM, Chen ST. Spinal epidural abscess successfully treated with percutaneous, computed tomography-guided, needle aspiration and parenteral antibiotic therapy: case report and review of the literature. Neurosurgery. 2002;51(2):509-512.

14. Perez-Toro MR, Burton AW, Hamid B, Koyyalagunta D. Twotuohy needle and catheter technique for fluoroscopically guided percutaneous drainage of spinal epidural abscess: a case report. Pain Med. 2009;10(3):501-505.

15. Schultz KD Jr, Comey CH, Haid RW Jr. Technical note. Pyogenic spinal epidural abscess: a minimally invasive technique for multisegmental decompression. J Spinal Disord. 2001;14(6): 546-549.

16. Panagiotopoulos V, Konstantinou D, Solomou E, et al. Extended cervicolumbar spinal epidural abscess associated with paraparesis successfully decompressed using a minimally invasive technique. Spine (Phila Pa 1976). 2004;29(14): E300-E303.

17. Lehman RA Jr, Lenke LG. Extensive epidural abscess treated with a thoracic laminoplasty. Spine J. 2011;11(8):798-799.

18. Lau D, Maa J, Mummaneni PV, Chou D. Holospinal epidural abscess. J Clin Neurosci. 2014;21(3):517-520.

19. Tahir MZ, Hassan RU, Enam SA. Management of an extensive spinal epidural abscess from $\mathrm{C}-1$ to the sacrum. Case report. J Neurosurg Spine. 2010;13(6):780-783.

20. Pola E, Rossi B, Nasto LA, et al. Surgical treatment of tuberculous spondylodiscitis. Eur Rev Med Pharmacol Sci. 2012;16(suppl 2):79-85.

21. Eroshkin A, Rainov N, Romanukha D. Surgical treatment of spondylodiscitis of the thoracic and lumbar spine. Austin Neurosurg Open Access. 2020;6(1):E1062.

22. Yuan S, Ma F, Wang Y, Gong P. Minimally invasive spine surgery in the treatment of pyogenic spondylodiscitis: an initial retrospective series study. Wideochir Inne Tech Malo Inwazyjne. 2019;14(2): 333-339.

\section{Disclosures}

The authors report no conflict of interest concerning the materials or methods used in this study or the findings specified in this paper.

\section{Author Contributions}

Conception and design: all authors. Acquisition of data: Romanukha. Analysis and interpretation of data: Romanukha, Voitsekhovskyi. Drafting the article: Romanukha. Critically revising the article: Eroshkin, Voitsekhovskyi. Statistical analysis: Voitsekhovskyi. Administrative, technical, and material support: Eroshkin. Study supervision: Eroshkin.

\section{Correspondence}

Dmytro Romanukha: Central Hospital of Ministry of Internal Affairs of Ukraine (Central Police Hospital), Kyiv, Ukraine. neuromanukha@ gmail.com. 